\title{
Incubation history prior to the canonical thermosensitive period determines sex in the American alligator
}

\author{
Jessica A McCoy ${ }^{*}$, Benjamin B Parrott*, Thomas R Rainwater ${ }^{1}$, Phillip M Wilkinson $^{2}$ and \\ Louis J Guillette Jr \\ Marine Biomedicine and Environmental Sciences Program, Hollings Marine Laboratory, Department of Obstetrics and \\ Gynecology, Medical University of South Carolina, 331 Fort Johnson Road, Charleston, South Carolina 29412, USA, \\ ${ }^{1}$ Baruch Institute of Coastal Ecology and Forest Science, Clemson University, Georgetown, South Carolina 29440, \\ USA and ${ }^{2}$ Tom Yawkey Wildlife Center, 407 Meeting Street, Georgetown, South Carolina 29440, USA
}

Correspondence should be addressed to B B Parrott; Email: benbparrott@gmail.com

*(J A McCoy and B B Parrott contributed equally to this work and are co-first authors)

\begin{abstract}
Despite the widespread occurrence of environmental sex determination (ESD) among vertebrates, our knowledge of the temporal dynamics by which environmental factors act on this process remains limited. In many reptiles, incubation temperature determines sex during a discrete developmental window just prior to and coincident with the differentiation of the gonads. Yet, there is substantial variation in sex ratios among different clutches of eggs incubated at identical temperatures during this period. Here, we test the hypothesis that temperatures experienced prior to the reported thermosensitive period for alligators (Alligator mississippiensis) can impact how the sex determination system responds to thermal cues later in development. Temperature shift experiments on eggs collected from the field within $24 \mathrm{~h}$ of oviposition were employed to decouple various maternal influences from thermal effects, and results demonstrate a previously undefined window of thermosensitivity occurring by stage 15 of embryonic development, six stages earlier than previously reported. We also examine the intrasexual expression of several male- and female-biased genes and show that while male-biased genes display no intrasexual differences, ovarian CYP19A1 (aromatase) transcript abundance differs by approximately twofold depending on thermal exposures experienced at early stages of embryonic development. These findings expand our understanding of the ESD in the alligator and provide the rationale for reevaluation of the temporal dynamics of sex determination in other crocodilians.

Reproduction (2015) 150 279-287
\end{abstract}

\section{Introduction}

Sex across a vast majority of metazoan taxa is bimodal, with organisms generally adopting either a male or female fate. However, belying this apparent simplicity is tremendous diversity in the mechanisms by which sex is initially determined. In some species, sex is determined at the moment of conception through the differential inheritance of sex chromosomes. Whereas in other taxa, the timing and mechanisms by which an individual acquires a specific sex remain much more ambiguous. Sex determination can be mediated by environmental factors alone, genetic elements, or some combination thereof that ultimately drives the trajectory of sexual development. The most evolutionary widespread mode of vertebrate environmental sex determination is temperature-dependent sex determination (TSD). It is widely accepted that during the development of TSD species, discrete temporal windows exist in which temperature influences the sex of an organism and are referred to as the thermosensitive period (TSP; Mrosovsky \& Pieau 1991, Pieau et al. 1999). Empirically defining the TSP in the context of concurrent developmental processes and oviposition is central to our understanding of the evolutionary and molecular underpinnings of TSD.

In all crocodilians studied to date, temperature is the only known determinant of sex. Ferguson \& Joanen (1982) first described TSD in crocodilians and demonstrated the lability of the alligator sex determination system. This study along with others established the pattern of TSD in Alligator mississippiensis along a gradient of increasing temperatures as female-malefemale (Lang \& Andrews 1994, Kohno et al. 2014). However, substantial variation in sex ratios among different clutches of eggs incubated at identical temperatures has been reported (Lang \& Andrews 1994, Rhen \& Lang 1998). Observations of 'clutch effects' on sex ratios are not unique to crocodilians and also occur in species 
of turtles and lizards (Janzen 1992, Bowden et al. 2000, Huang et al. 2008, Kratochvil et al. 2008) with TSD. However, the underlying explanations for these widely observed clutch effects are not clear.

The TSP in all reptiles displaying TSD is thought to encompass the initial developmental stages in which the trajectory of the bipotential gonad bifurcates, resulting in either a testis or an ovary (Pieau \& Dorizzi 1981, Bull 1987, Wibbels et al. 1991, Lang \& Andrews 1994, Shine et al. 2007). After the sexual dimorphic differentiation of the gonad progresses beyond a specific developmental threshold, the sex of that individual is irreversibly established (Deeming \& Ferguson 1988, Wibbels et al. 1991). However, the specific period and associated developmental stages that characterize the figurative opening of the TSP remain ambiguous.

Our knowledge regarding the temporal and developmental features of thermosensitivity in alligators originates from experimental designs that incorporate the incubation of embryos at male- and female-producing temperatures (MPT and FPT respectively). Initial reports utilizing singleshift designs reported different periods of thermosensitivity depending on the original incubation temperature; however, the details regarding observed sex ratios are not included in the report, making interpretation difficult (Ferguson \& Joanen 1982, 1983). With double-shift designs, the investigator examines how sex ratios are affected by temporary pulses of an MPT against the background of an FPT and vice versa during different developmental periods. Lang \& Andrews (1994) applied both single- and doubletemperature shift experiments to refine the period of thermosensitivity in A. mississippiensis and demonstrated that, irrespective of incubation temperature, a discrete TSP occurs from embryonic stages 21 to 24 ( $\sim 30-45$ days of incubation) and encompasses the period of gonadal differentiation. The authors attributed the reported discrepancies between their findings and those reported previously to thermal features of the shift regimes including the magnitude of the temperature shift and the use of a MPT $\left(34{ }^{\circ} \mathrm{C}\right)$ that they recognized to produce both males and females (Lang \& Andrews 1994). Since the publication of this report, the period of development between embryonic stages 21 and 24 has served as the canonical period of thermosensitivity in alligator, and numerous studies have incorporated this specific period into designs aimed at characterizing the origins and mechanisms of TSD (Conley et al. 1997, Western et al. 1999a, Gabriel et al. 2001, Milnes et al. 2002, Parrott et al. 2014).

Here, we present a thermal shift design to investigate specifically the impact of early embryonic thermal exposures (prior to stage 19) on resulting sex ratios. The rationale for this experiment originates from previous observations by our group suggesting a link between incubation temperatures in early development and resulting sex ratios. In the current study we follow up this initial observation with a temperature shift experiment aimed at disentangling the influence of 'early' incubation temperature from other confounding factors associated with clutch (i.e., genetic, epigenetic, and maternally deposited factors). We went to great lengths to collect alligator eggs directly after oviposition and use these eggs to clearly define a window of thermosensitivity occurring earlier in development than previously realized. Furthermore, we consider whether the temporal dynamics of exposure to a feminizing temperature impact the expression of gonadal genes. Together, these findings contribute to our understanding of thermosensitivity in crocodilians and provide insight for future investigations aimed at clarifying the proximate mechanisms controlling sex differentiation in species that display TSD.

\section{Materials and methods}

\section{Field collections and egg incubations}

The Institutional Animal Care and Use Committee at the Medical University of South Carolina approved all of the experiments reported here (protocol AR \#3036). The Florida Fish and Wildlife Conservation Commission, U.S. Fish and Wildlife Service, and South Carolina Department of Natural Resources provided permits for field collections. For all of the experiments, eggs were incubated in industrial bus pans containing damp sphagnum moss and the temperature was monitored in 10-min increments with multiple temperature data loggers (Onset HOBO Data Loggers, Bourne, MA, USA) placed in each bin. Controlled incubations took place in commercial incubators (Thermo Scientific, Waltham, MA, USA, Forma Environmental Chambers, model \#3920).

The current thermal shift study was prompted by results from a prior experiment, which was designed to address a different question regarding the levels of intra-sexual variation in various traits. For that initial experiment, all of the eggs were collected on the same day from Lake Apopka, FL, USA. Eggs representing eight clutches were transported from the field to our laboratory and one embryo from each clutch was necropsied to ascertain the developmental stage (Ferguson 1985). All of the eggs were subsequently incubated at $30^{\circ} \mathrm{C}$ until reaching stage 19 . Then, eggs from each clutch were distributed among four different incubation temperatures $\left(30,32,33.5\right.$, and $\left.34.5^{\circ} \mathrm{C}\right)$ until stage 27.5. Eggs were then transferred to $30^{\circ} \mathrm{C}$ until hatch. At 1 week of age, hatchlings were necropsied and gonads were preserved in RNAlater.

For the current study, seven clutches of eggs were collected within 24 h of oviposition from the Tom Yawkey Wildlife Center and Heritage Preserve (TYWCHP) near Georgetown, SC, USA, and transported to the laboratory. Nests were identified during weekly helicopter flights over the TYWCHP during the nesting season, and nests were subsequently visited daily on foot. If nests did not yet contain eggs, they were visited every morning until the female laid eggs. Therefore, it was possible to ensure that only those clutches that were oviposited within the previous $24 \mathrm{~h}$ were collected for laboratory incubations. Immediately on arrival at the laboratory, eggs from each clutch were distributed across six incubation regimens (Experimental groups A-F). The treatment groups were designed to test whether temperatures 
Table 1 Distribution of each clutch across the six treatment groups. The number of surviving embryos comprising each treatment group is noted as the proportion of males.

\begin{tabular}{|c|c|c|c|c|c|c|c|}
\hline Clutch & A & B & C & D & $\mathbf{E}$ & $\mathbf{F}$ & Total \\
\hline 3 & $4 / 4$ & $0 / 1$ & $1 / 1$ & $0 / 0$ & $0 / 0$ & $0 / 1$ & 7 \\
\hline 4 & $5 / 5$ & $0 / 3$ & $0 / 1$ & $0 / 6$ & $6 / 6$ & $0 / 5$ & 26 \\
\hline 6 & $4 / 4$ & $1 / 3$ & $6 / 6$ & $0 / 3$ & $3 / 3$ & $0 / 3$ & 22 \\
\hline 10 & $3 / 3$ & $0 / 2$ & $4 / 4$ & $0 / 5$ & $3 / 3$ & $0 / 3$ & 20 \\
\hline 16 & $3 / 3$ & $0 / 4$ & $7 / 7$ & $0 / 3$ & $4 / 4$ & $0 / 2$ & 23 \\
\hline 20 & $4 / 5$ & $0 / 5$ & $6 / 6$ & $0 / 3$ & $5 / 5$ & $0 / 2$ & 26 \\
\hline 21 & $4 / 4$ & $0 / 2$ & $5 / 5$ & $0 / 3$ & $2 / 3$ & $0 / 0$ & 17 \\
\hline Total & $27 / 28$ & $1 / 20$ & $29 / 30$ & $0 / 23$ & $23 / 24$ & $0 / 16$ & 141 \\
\hline
\end{tabular}

experienced prior to the canonical TSP (stages 21-24) influence sex determination and, if so, to specify the developmental time frame that displays thermosensitivity. Each treatment group originally included representatives from each of the seven clutches. However, due to embryo mortality, two clutches (clutches 3 and 21) were not represented in every treatment group. The number and sex of viable embryos from each clutch across treatment groups is shown in Table 1. To calculate egg transfer and final necropsy dates, a subset of eggs were necropsied at various points during development, and embryonic stage was determined (Ferguson 1985, Kohno \& Guillette 2013). All of the embryos were necropsied at developmental stage 27 and gonadal sex was determined by quantitative real-time PCR (qPCR).

\section{QPCR to sex embryos and quantify gonadal gene expression}

Sex was determined by assessing the levels of two transcripts, anti-Müllerian hormone $(A M H)$ and $C Y P 19 A 1$. Expression levels of these transcripts display a clear bimodal distribution in regards to sexual dimorphism and differ between males and females by orders of magnitude. Validation of this molecular sex assessment indicates that there is a $98.7 \%(213 / 216)$ concordance between histology-based sex assignment and that based on expression patterns of sexually dimorphic genes (Kohno et al. 2015). RNA isolation, purification, and RT were conducted as previously reported (Kohno et al. 2010). Expression levels were determined by comparison to plasmid DNA standard curves that contained known copies of each target gene amplicon. To ensure consistency, standard curves were run on each plate containing unknowns. Each sample, including unknowns and those belonging to standard curves, were run in triplicate and mean values were taken to represent each sample. A mean copy number of each target gene within each sample was subsequently normalized to the number of copies of control gene, ribosomal protein L8 ( $R P L 8)$. Complete concordance was observed in the sex assignment indicated by the results of the qPCR analysis of $A M H$ and CYP19A1. To further address potential links between thermal exposures during embryonic development and transcriptional activity of stage 27 gonads, we expanded the qPCR analysis to also include SOX9 and FOXL2.

\section{Statistical analyses}

All statistical analyses were performed with SPSS Statistics version 22 (IBM, Armonk, NY, USA) and GraphPad Prism version 6.01 (GraphPad Software, La Jolla, CA, USA). For the initial experiment, linear regression and a sigmoidal dose response (variable slope) analyses were utilized to assess the relationship between sex ratio and incubation history. In the present experiment, the influences of temperature treatment and clutch on sex ratios were tested using a binary logistic regression analyses. A two-way ANOVA incorporating clutch as a random effect and a Tukey's multiple comparison post-hoc test were used to determine whether differences in transcript abundance differed within one sex among different temperature treatment groups. A linear regression analysis was used to determine the relationship between CYP19A1 and FOXL2. Statistical significance was set at $P<0.05$ for all tests.

\section{Results}

The impetus for the design of the current experiment stemmed from a previous experiment that was originally developed to address the relationship between temperatures experienced from stages 19 to 27 and intra-sexual characteristics. Given that this previous design involved the collection of eggs from nest sites at different stages of embryonic development, the duration of time they spent at the laboratory holding temperature $\left(30^{\circ} \mathrm{C}\right)$ prior to reaching stage 19 also varied. We hypothesized, on the basis of observed sex ratios, that variations in the incubation temperatures experienced prior to embryonic stage 19 might influence how individual clutches respond to thermal signals later in development. Support for this prediction was bolstered with linear regression analyses that show a strong correlation between the length of time individual clutches were incubated at the holding temperature $\left(30^{\circ} \mathrm{C}\right)$ prior to the TSP and the sex ratios resulting from incubations at transitional range temperatures that produce mixed sex ratios $(n=8$ clutches, $P=0.02, R^{2}=0.60$, Fig. 1). Further, a sigmoidal dose-response analysis revealed a model with a remarkably good fit to the data $\left(R^{2}=0.93\right.$, dotted line in Fig. 1). However, due to the limitations of the design, we were unable to separate influences of incubation temperature occurring before the canonical TSP from other influences (e.g., genetic, epigenetic, and maternally deposited factors) associated with clutch. The present follow-up experiment was designed to test the hypothesis that early incubation temperatures influence 


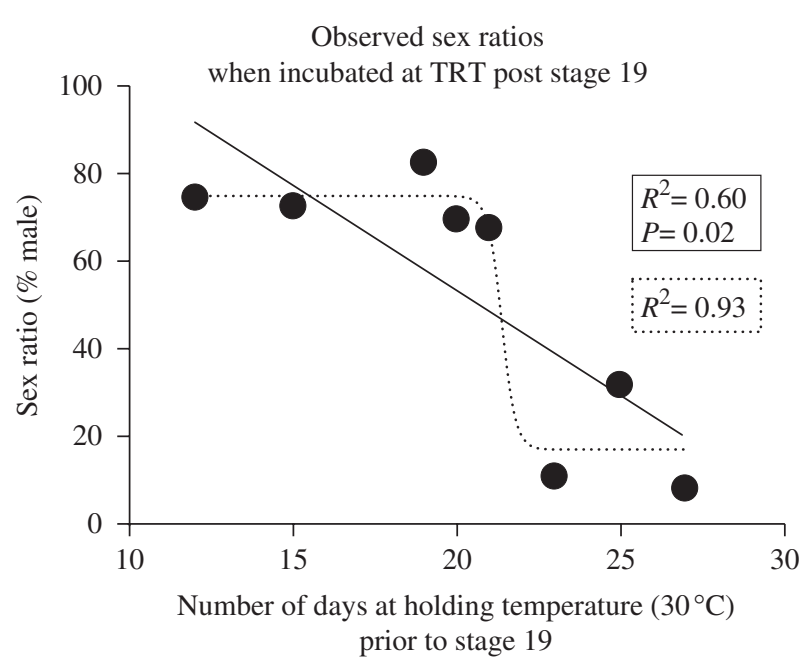

Figure 1 The current experimental approach was prompted by results from a previous study suggesting that conditions experienced during early embryonic development might influence alligator sex determination. Analyses of sex ratios resulting from incubation at transitional range temperatures (TRT; post stage 19) reveal clutchrelated variation in the sex determination response. The original design involved the collection of eggs from the field and subsequent incubation at a holding temperature $\left(30^{\circ} \mathrm{C}\right)$ until reaching stage 19 of development. Because all eggs were collected from the field at different stages of embryonic development, the duration of time they spent at the holding temperature also varied. A relationship between this feature of the early incubation and obtained sex ratios is observed.

sex determination in alligators and further define putative 'early' window(s) of thermosensitivity.

To test the early incubation temperature hypothesis, the present experiment involved the collection of eggs from field sites within $24 \mathrm{~h}$ of oviposition and subsequent laboratory incubation with pulses of $30{ }^{\circ} \mathrm{C}$ against a background temperature of $32.5^{\circ} \mathrm{C}$ (Fig. 2). A binary logistic regression analysis was performed to ascertain the effects of temperature-incubation treatment and clutch on sex ratios. The model was statistically significant $\left(\chi^{2}=162.084, P<0.001\right)$, explained $91.2 \%$ (Nagelkerke $R^{2}$ ) of the variance in sex ratios, and correctly classified $97.2 \%$ of the cases. While the effect of the temperature treatment group was significant $(P<0.001)$, the effect of clutch was not $(P=0.31)$. Constant incubation of eggs collected directly after oviposition at $32.5{ }^{\circ} \mathrm{C}$ from stages 1 to 27 resulted in the production of $96 \%$ males (treatment group $\mathrm{A}, n=28$, Table 2). Surprisingly, incubating eggs at $30{ }^{\circ} \mathrm{C}$ from stages 1 to 19 resulted in the production of $95 \%$ females (treatment group B, $n=20$, Table 2), demonstrating a dramatic influence of temperature on sex determination occurring well prior to the previously established TSP. Further, the observed effect was not reversed by subsequent exposure to temperatures producing almost exclusively males. Incubating eggs at $30{ }^{\circ} \mathrm{C}$ from stages 1 to 9 or from stages 4 to 15 had no effect on sex ratios when compared to treatment group A (treatment group C, $n=30$ and treatment group $\mathrm{E}, n=24$, respectively; Table 2). Conversely, incubating eggs at $30{ }^{\circ} \mathrm{C}$ during stages 9-19 was sufficient to override the malepromoting effects of incubations at $32.5^{\circ} \mathrm{C}$ (treatment group $\mathrm{D}, n=23$, Table 2). Together, these data demonstrate that by stage 15 and potentially earlier, sex determination in the alligator displays thermosensitivity and, further, that these thermal influences acting on early developmental processes are stable.

To further investigate the interactions between the embryonic timing of thermal exposures and sex determination in a TSD species, we tested whether individuals of the same sex, but resulting from different thermal treatments, displayed variation in the expression patterns of genes with key roles in gonadal function.

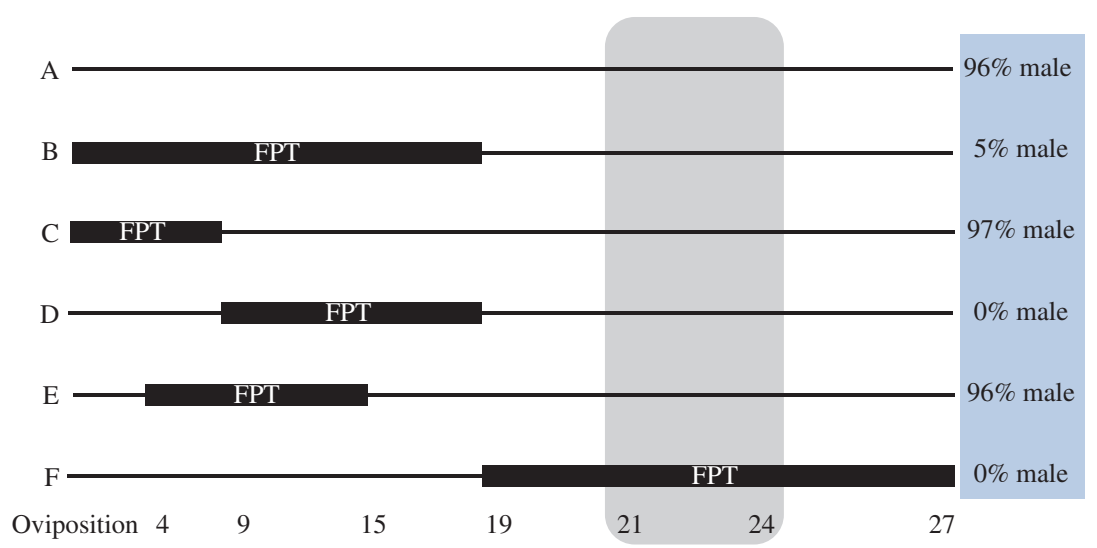

Figure 2 Thermal shift designs and resulting sex ratios. Letters on the left side of the panel denote the temperature treatment group and numbers along the $X$-axis indicate developmental stage. Details for each treatment group are included in Table 1 . The period during which eggs were exposed to a female-producing temperature $\left(\mathrm{FPT}\right.$; set $=30^{\circ} \mathrm{C}$, actual $\left.=29.3 \pm 0.4{ }^{\circ} \mathrm{C}\right)$ is denoted with a thickened black line. Outside of this period, the eggs were exposed to a temperature predicted to yield mixed sex ratios (set $=32.5^{\circ} \mathrm{C}$, actual $32.6 \pm 0.2{ }^{\circ} \mathrm{C}$ ). The observed sex ratios (percent male) are listed on the right side of the panel. The shaded area that encompasses stage 21 through stage 24 highlights the thermosensitive period (TSP) previously reported for the American alligator. 
Table 2 Incubation conditions and sex ratios obtained from temperature shift experiments. For each incubation group, the duration of exposure to a female-producing temperature $\left(\mathrm{set}=30^{\circ} \mathrm{C}\right.$, actual $=29.3 \pm 0.4{ }^{\circ} \mathrm{C}$ ) is indicated with respect to both developmental stage and days of incubation. Outside of this window, the egg was held at a temperature producing mostly males (set $=32.5{ }^{\circ} \mathrm{C}$, actual $32.6 \pm 0.2{ }^{\circ} \mathrm{C}$ ). The number of males and females that developed from each condition along with observed sex ratios are reported.

\begin{tabular}{|c|c|c|c|c|c|c|c|c|}
\hline $\begin{array}{l}\text { Treatment } \\
\text { group }\end{array}$ & $\begin{array}{l}\text { FPT incubation } \\
\text { (stage) }\end{array}$ & $\begin{array}{l}\text { FPT incubation } \\
\text { (days) }\end{array}$ & Days FPT $(n)$ & Total eggs & Males $(n)$ & Females $(n)$ & Males (\%) & Females $(\%)$ \\
\hline A & NA & NA & NA & 28 & 27 & 1 & 96 & 4 \\
\hline B & $1-19$ & $1-27$ & 26 & 20 & 1 & 19 & 5 & 95 \\
\hline C & $1-9$ & $1-9$ & 8 & 30 & 29 & 1 & 97 & 3 \\
\hline D & 9-19 & $9-26$ & 18 & 23 & 0 & 23 & 0 & 100 \\
\hline E & $4-15$ & $4-17$ & 14 & 24 & 23 & 1 & 96 & 4 \\
\hline $\mathrm{F}$ & $19-27$ & $23-60$ & 38 & 16 & 0 & 16 & 0 & 100 \\
\hline
\end{tabular}

We first examined male-biased genes. For those thermal regimens that produced primarily males (treatment groups $\mathrm{A}, \mathrm{C}$, and $\mathrm{E}$ ) and those that produced primarily females (treatment groups $\mathrm{B}, \mathrm{D}$, and $\mathrm{F}$ ), the transcript levels of SOX9 and $A M H$ were quantified (Fig. 3). Both SOX9 and $A M H$ are upstream effectors in the testisdetermining pathway with roles in Sertoli cell differentiation and reproductive tract development respectively (Tran et al. 1977, Morais da Silva et al. 1996, Western et al. 1999a,b). Analysis reveals equal abundance of SOX9 and $A M H$ across treatments, suggesting that varying the timing and duration of a 'dose' with feminizing temperature does not impact $A M H$ and SOX9 transcriptional activity in the testis or ovary at embryonic stage 27 (Fig. 3).

For treatments that produced primarily females (treatment groups $\mathrm{B}, \mathrm{D}$, and $\mathrm{F}$ ), we assessed transcript levels of FOXL2 and CYP19A1, genes with central roles in ovarian differentiation (Ditewig \& Yao 2005, Ottolenghi et al. 2007; Fig. 4). For these genes, we were not able to quantify the very low levels of FOXL2 and CYP19A1 in testes, given that $90 \%(72 / 80)$ and $100 \%(80 / 80)$ of individual testis samples fell below the level of quantification for CYP19A1 and FOXL2 respectively. In those eight testis samples in which CYP19A1 expression could be measured, the average normalized expression values were three orders of magnitude less than those measured in ovarian samples $\left(5.3 \times 10^{-5} \pm 2.9 \times 10^{-5}\right.$ copies/ $\mu \mathrm{l}, n=8$ ). A two-way ANOVA examining the effect of temperature incubation treatment and clutch on ovarian CYP19A1 expression revealed significant variation that could be attributed to thermal exposures, with treatment group $\mathrm{F}$ displaying nearly double the expression level of CYP19A1 compared to treatment groups $B$ and D (Fig. 4C; $P=0.02, F=5.625$ ). An effect of clutch was not detected $(P=0.17, F=1.879)$ nor was an interaction observed between clutch and temperature incubation treatment $(P=0.124, F=1.667)$. FOXL2, a conserved transcription factor thought to directly drive CYP19A1 transcription in mammals, was also analyzed (Pannetier et al. 2006, Georges et al. 2014; Fig. 4B). Regression analysis with FOXL2 and CYP19A1 ovarian transcript abundance reveals a positive linear relationship, supporting the presence of a similar regulatory process in alligator (Fig. 4D; $R^{2}=0.3$, $P<0.0001)$. While the pattern of FOXL2 expression in response to different thermal regimens resembles that of
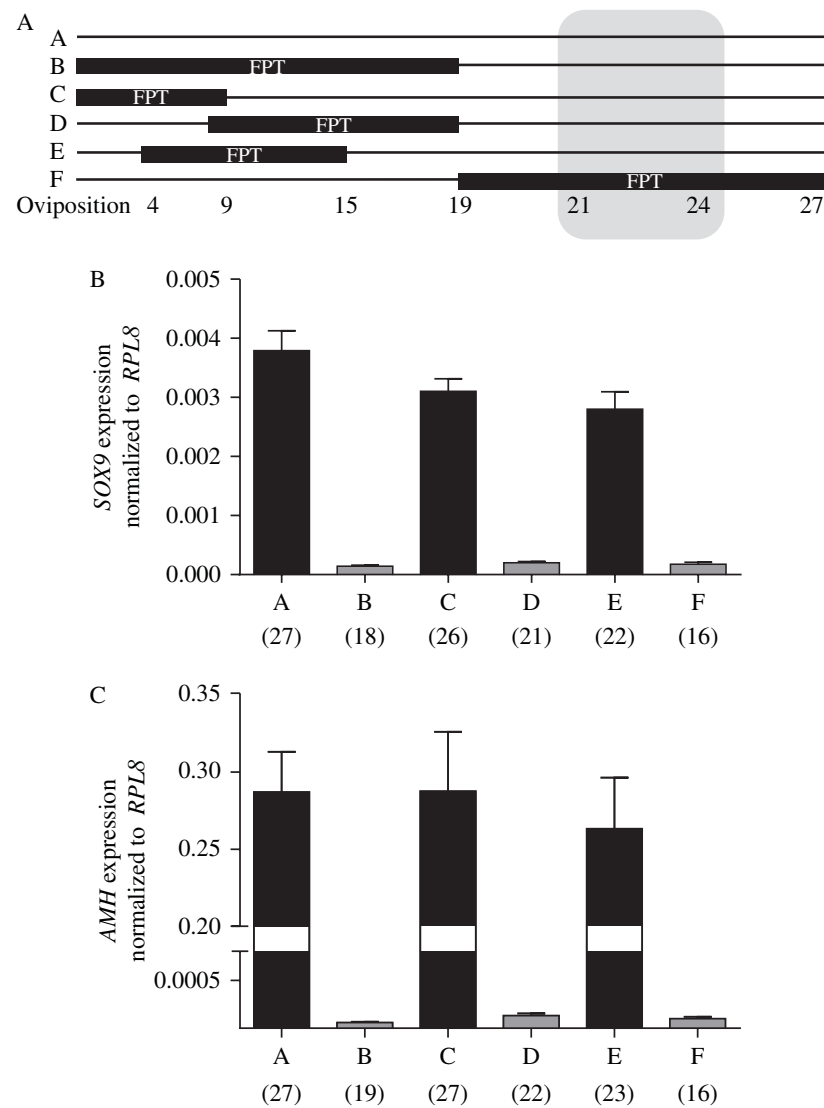

Figure 3 Gonadal expression of male-biased genes with key roles in sex differentiation in stage 27 embryos. The transcript abundance of malebiased genes was determined for all treatment groups (A). Testis (black bars; groups $\mathrm{A}, \mathrm{C}$, and $\mathrm{E}$ ) and ovarian (gray bars; groups $\mathrm{B}, \mathrm{D}$, and $\mathrm{F}$ ) levels of $S O X 9(\mathrm{~B})$ and $A M H(C)$ transcripts were quantified using qPCR. A two-way ANOVA revealed no statistically significant differences among males or females. The sample size for each group is included in parentheses and the error bars represent the S.E.M. for each treatment group. The shaded area that encompasses stage 21 through stage 24 (A) highlights the thermosensitive period (TSP) previously reported for the American alligator. 
A
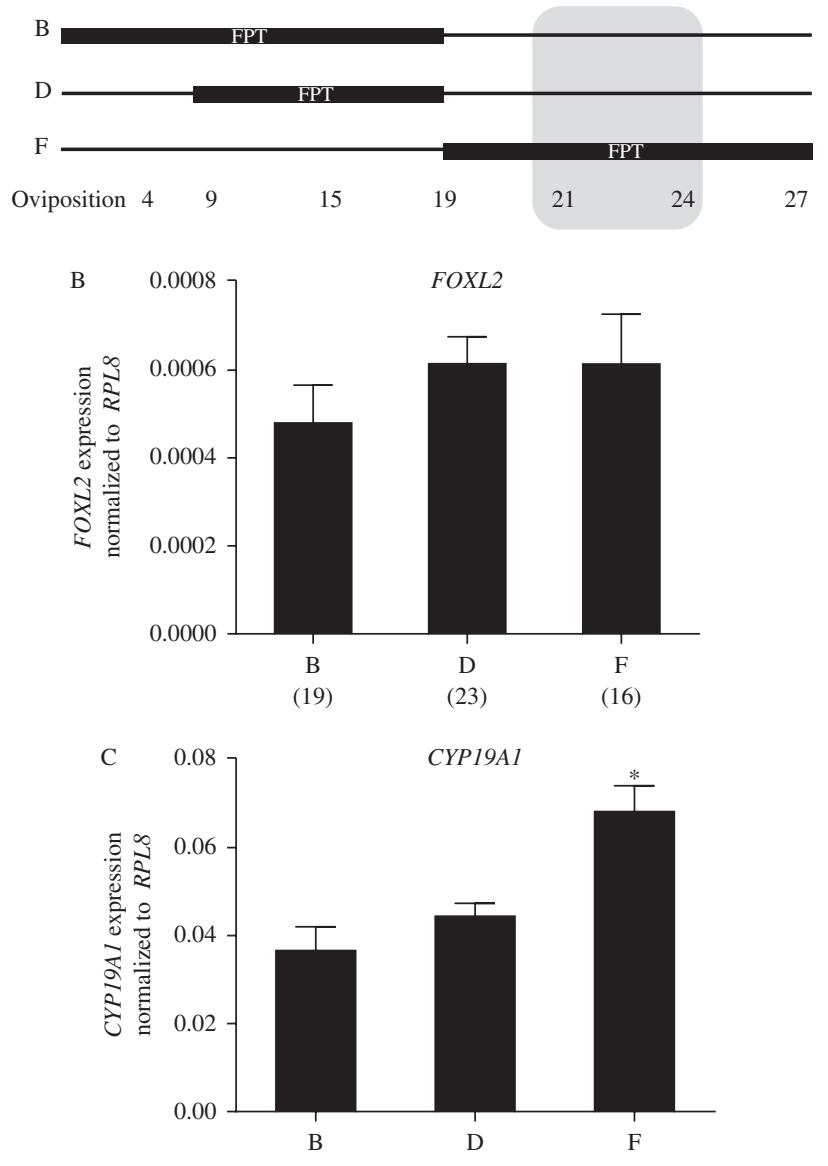

(19)

(23)



Figure 4 Gonadal expression of female-biased genes involved in determining ovarian fate and differentiation in stage 27 embryos. For treatment groups producing primarily females (A), ovarian levels of FOXL2 (B), and CYP19A1 (C) were assessed using qPCR. Because the abundance of FOXL2 and CYP19A1 in testis was below the level of quantification for this analysis, expression levels from groups that produced primarily males are not reported here (groups $A, C$, and E). Results of a two-way ANOVA are shown; ${ }^{*} P<0.05$. The sample size for each group is included in parentheses, and the error bars represent the S.E.M. for each treatment group. (D) A positive linear relationship was identified between ovarian FOXL2 and CYP19A1 transcript abundance $\left(R^{2}=0.3, P<0.0001\right)$. The shaded area that encompasses stage 21 through stage $24(\mathrm{~A})$ highlights the thermosensitive period (TSP) previously reported for the American alligator.
CYP19A1, the difference between treatment groups does not vary significantly (Fig. $4 \mathrm{~B} ; P=0.91$ ). Together these observations provide additional insights into the dynamics of TSD and provide substance for future inquiry regarding the proximate mechanisms linking temperature to the molecular and cellular cascade that dictates the trajectory of sexual development.

\section{Discussion}

Here, we report sex ratios for alligators resulting from incubations at different temperatures throughout embryonic development and show that incubation temperature experienced during early development profoundly affects sex ratios. Interestingly, these feminizing effects on sex ratios were not overridden by subsequent exposure to temperatures that normally produce males, suggesting a stable and persistent effect of thermal influences experienced during early development. Incubating at $30^{\circ} \mathrm{C}$ from stages 1 to 19 against an otherwise MPT resulted in all females. When shorter pulses of feminizing temperature during these early stages were tested, only a pulse from stages 9 to 19 was able to recapitulate this feminizing effect, whereas pulses from stages 1 to 9 and 4 to 15 had no effect. Given the resolution these shift experiments provide, it is most likely that the TSP begins by stage 15, if not earlier in development. However, additional shift experiments that vary in stage and duration are needed to more precisely define the opening of the alligator TSP. Further, these pulses during early development were similar but not identical in duration, and thus, we cannot rule out that longer pulses (e.g., from stages 1 to 12) of feminizing temperatures might reveal earlier thermosensitivity not detected in this study. In addition, it should be noted that sensitivity might not be uniformly distributed across the TSP and that particular developmental stages could display heightened thermosensitivity relative to others. These results uncover a window of sensitivity to incubation temperature that occurs well prior to the previously known TSP and should provide important context for future studies aimed at examining the molecular, ecological, and evolutionary dynamics of TSD (Lang \& Andrews 1994).

The dissimilarities between the timing of the TSP in alligator reported here compared to that reported by Lang \& Andrews (1994) might result from key differences in egg incubation designs. In the current study, incubation conditions were tightly controlled starting at $24 \mathrm{~h}$ post-oviposition. In the Lang \& Andrews (1994) design, eggs were harvested within a week of oviposition, a feature that resulted in variable early incubation conditions of eggs incorporated into their single- and double-shift designs. Other differences between the two studies include the temperatures used for incubating eggs, sexing methods, and specific timing of shifts. For these reasons and those mentioned in the 
preceding paragraph (i.e., different degrees of thermal sensitivity across stages), an extraordinary number of eggs and resources would be necessary to sufficiently test the thermosensitivity of sex determination in regards to every combination of developmental stage, length of thermal exposure, and degree of temperature. Consequently, it is easier to demonstrate that a particular developmental stage shows thermosensitivity under certain conditions rather than show an absolute absence of thermosensitivity. Thus, the findings reported here build on, rather than overturn, previous seminal studies examining the TSP in alligators.

Our findings suggest that sex determination is thermosensitive by stage 15 . Further studies aimed at determining the biological processes that demarcate the commencement of temperature sensitivity are needed to expound the underpinnings of TSD. Sexually dimorphic development of the gonad is first observed at the histological level at or just after stage 21 and consists of an enlargement of presumptive pre-Sertoli cells within the medulla (Smith \& Joss 1993). Sexually dimorphic gonadal gene expression is first detected at approximately stage 22 (Western et al. 1999b). Thus, our findings indicate that the thermosensitivity of sex determination commences substantially earlier than the onset of sexual dimorphism within the gonad. This initial portion of the TSP occurring before the onset of sexually dimorphic differentiation is more consistent with TSPs reported in turtle species, where thermosensitivity is observed prior to the sexually dimorphic gonadal differentiation and continues until shortly after this dimorphism is established (Bull \& Vogt 1981, Pieau \& Dorizzi 1981, Wibbels et al. 1991). Recent reports in turtles and alligators have demonstrated a role for epigenetic patterning in coordinating sexually dimorphic gene expression (Matsumoto et al. 2013, Parrott et al. 2014). The finding that the effects on sex ratios resulting from pulses of $\mathrm{FPT}$, occurring prior to the onset of histological and transcriptional sexual dimorphism, were not reversed by subsequent incubations provides insights into the stability of the mechanisms underlying this early thermosensitivity. It is tempting to speculate that this early thermosensitivity might be mediated by epigenetic modifications to the genome that influence the trajectories of downstream developmental processes. Studies aimed at characterizing the gonadal epigenome, prior to the onset of sexually dimorphic gene expression, in terms of DNA methylation patterns, histone modifications, and noncoding RNAs should provide insights into the molecular nature of thermosensitivity in TSD species.

The pivotal role of gonadal hormones in the establishment and maintenance of sexual dimorphism is well documented (Meyer et al. 1975, McEwen et al. 1977, Andersson et al. 1991). However, the environmental and molecular origins within sex variation are unclear. Our finding linking the magnitude of female-biased transcripts to the pattern of thermal exposure during embryonic development is especially intriguing in light of this latter question. Ovarian levels of aromatase, the enzyme responsible for the biosynthesis of estrogen from androgens, vary considerably depending on the developmental timing of exposure to a feminizing temperature $\left(30^{\circ} \mathrm{C}\right)$. FOXL2, a member of the forkhead transcription factor family, promotes CYP19A1 transcription in both mammalian and non-mammalian species through direct interaction with the CYP19A1 ovarian specific promoter (Govoroun et al. 2004, Pannetier et al. 2006, Okada et al. 2009). A comparison of FOXL2 levels among the females produced from different incubation treatments reveals a pattern that lacks statistical significance but is consistent with a role for FOXL2 in positively regulating CYP19A1 transcription. It is conceivable that even small changes in transcription factor abundance can exert significant influences on transcript levels of target genes (i.e., CYP19A1). The variation embedded within qPCR methodology could prevent detection of these small, but biological relevant, changes in the levels of upstream regulatory factors. Given the critical roles of steroid hormones during development, any alterations to factors involved in their biosynthesis have the potential to influence organizational events during embryogenesis and could provide a source of variation across a wide range of secondary sex traits later in life (Bergeron et al. 1998, Pointer et al. 2013). The CYP19A1 expression differences identified in this study provide strong rationale for future studies aimed at further characterizing how the patterns of thermal exposures experienced during embryogenesis contribute to molecular, physiological, and behavioral differences among individuals of the same sex.

Finally, the most popular model explaining the adaptive significance of TSD is the Charnov-Bull model (Charnov \& Bull 1977). In this model, TSD provides selective advantages over genetic sex determination when the fitness of individuals from each sex is differentially affected by incubations at varying temperatures. The TSP described by Lang \& Andrews (1994) occurs later in development relative to those TSPs described for tuatara and turtles, which generally occur during the second quarter of development (Ewert et al. 2004, Mitchell et al. 2006, Nelson et al. 2010). In those lizard species studied, the TSP roughly encompasses the middle-third of development post-oviposition. Owing to the late timing of the previously reported TSP relative to oviposition, crocodilians have been portrayed as an example in which mothers lack predictive power regarding incubation temperatures during the TSP (Shine et al. 2007). In light of the results reported here, perhaps the effects of maternal influences on post-oviposition incubation factors are stronger than previously realized and should be considered in future models. 


\section{Declaration of interest}

The authors declare that there is no conflict of interest that could be perceived as prejudicing the impartiality of the research reported.

\section{Funding}

This work was supported by the Center of Economic Excellence Center for Marine Genomics (L J Guillette Jr).

\section{Acknowledgements}

We very much appreciate the efforts of members of the Florida Fish and Wildlife Conservation (FWC) alligator team, Allan Woodward, Arnold Brunell, Cameron Carter, and Patrick Delaney, as well as Matthew Guillette (Medical University of South Carolina) and Jamie Dozier of the South Carolina Department of Natural Resources (DNR) and Tom Yawkey Wildlife Center for continued field support. We also thank Brenna Doheny and other members of the Guillette Laboratory for helpful discussions and insightful comments regarding the manuscript. In addition, we would also like to thank an anonymous reviewer whose suggestions regarding the 'Discussion' significantly enhanced the manuscript.

\section{References}

Andersson S, Berman DM, Jenkins EP \& Russell DW 1991 Deletion of steroid $5 \alpha$-reductase 2 gene in male pseudohermaphroditism. Nature 354 159-161. (doi:10.1038/354159a0)

Bergeron JM, Gahr M, Horan K, Wibbels T \& Crews D 1998 Cloning and in situ hybridization analysis of estrogen receptor in the developing gonad of the red-eared slider turtle, a specied with temperature-dependent sex determination. Development, Growth \& Differentiation 40 243-254. (doi:10.1046/j.1440-169X.1998.00013.x)

Bowden RM, Ewert MA \& Nelson CE 2000 Environmental sex determination in a reptile varies seasonally and with yolk hormones. Philosophical Transactions of the Royal Society of London. Series B, Biological Sciences 267 1745-1749. (doi:10.1098/rspb.2000.1205)

Bull J 1987 Temperature-sensitive periods of sex determination in a lizard: similarities with turtles and crocodiles. Journal of Experimental Zoology 241 143-148. (doi:10.1002/jez.1402410118)

Bull JJ \& Vogt RC 1981 Temperature-sensitive periods of sex determination in emydid turtles. Journal of Experimental Zoology 218 435-440. (doi:10.1002/jez.1402180315)

Charnov EL \& Bull JJ 1977 When is sex environmentally determined? Nature 266 828-830. (doi:10.1038/266828a0)

Conley AJ, Elf P, Corbin CJ, Dubowsky S, Fivizzani A \& Lang JW 1997 Yolk steroids decline during sexual differentiation in the alligator. General and Comparative Endocrinology 107 191-200. (doi:10.1006/gcen.1997.6913)

Deeming DC \& Ferguson MW 1988 Environmental regulation of sex determination in reptiles. Philosophical Transactions of the Royal Society of London. Series B, Biological Sciences 322 19-39. (doi:10.1098/rstb. 1988.0111)

Ditewig AC \& Yao HH 2005 Organogenesis of the ovary: a comparative review on vertebrate ovary formation. Organogenesis 2 36-41. (doi:10. 4161/org.2.2.2491)

Ewert MA, Etchberger CR \& Nelson CE 2004 Turtle sex-determining modes and TSD patterns, and some TSD pattern correlates. In TemperatureDependent Sex Determination in Vertebrates, ch 3, pp 21-32. Eds N Valenzuela \& V Lance. Smithsonian Books. Washington D.C.

Ferguson MWJ 1985 Reproductive biology and embryology of the crocodilians. In Biology of the Reptilia, pp 329-491. Eds C Gans, FS Billet, PFA Maderson. John Wiley and Sons, New York, New York, USA.
Ferguson MWJ \& Joanen T 1982 Temperature of egg incubation determines sex in Alligator mississippiensis. Nature 296 850-852. (doi:10.1038/ 296850a0)

Ferguson MWJ \& Joanen T 1983 Temperature-dependent sex determination in Alligator mississippiensis. Journal of Zoology 200 143-177. (doi:10. 1111/j.1469-7998.1983.tb05781.x)

Gabriel WN, Blumberg B, Sutton S, Place AR \& Lance VA 2001 Alligator aromatase cDNA sequence and its expression in embryos at male and female incubation temperatures. Journal of Experimental Zoology 290 439-448. (doi:10.1002/jez.1087)

Georges A, Auguste A, Bessiere L, Vanet A, Todeschini AL \& Veitia RA 2014 FOXL2: a central transcription factor of the ovary. Journal of Molecular Endocrinology 52 R17-R33. (doi:10.1530/JME-13-0159)

Govoroun MS, Pannetier M, Pailhoux E, Cocquet J, Brillard JP, Couty I, Batellier F \& Cotinot C 2004 Isolation of chicken homolog of the FOXL2 gene and comparison of its expression patterns with those of aromatase during ovarian development. Developmental Dynamics 231 859-870. (doi:10.1002/dvdy.20189)

Huang V, Sakata JT, Rhen T, Coomber P, Simmonds S \& Crews D 2008 Constraints on temperature-dependent sex determination in the leopard gecko (Eublepharis macularius): response to Kratochvil et al. Die Naturwissenschaften 95 1137-1142. (doi:10.1007/s00114-0080433-5)

Janzen FJ 1992 Heritable variation for sex ratio under environmental sex determination in the common snapping turtle (Chelydra serpentina). Genetics 131 155-161.

Kohno S \& Guillette LJ Jr 2013 Endocrine disruption and reptiles: using the unique attributes of temperature-dependent sex determination to assess impacts. Ed P Matthiessen, John Wiley \& Sons, Inc., Hoboken, NJ, USA. (pp 245-271). (doi:10.1002/9781118355961.ch9)

Kohno S, Katsu Y, Urushitani H, Ohta Y, Iguchi T \& Guillette JL Jr 2010 Potential contributions of heat shock proteins to temperature-dependent sex determination in the American alligator. Sexual Development 4 73-87. (doi:10.1159/000260374)

Kohno S, Parrott BB, Yatsu R, Miyagawa S, Moore BC, Iguchi T \& Guillette LJ Jr 2014 Gonadal differentiation in reptiles exhibiting environmental sex determination. Sexual Development 8 208-226. (doi:10.1159/000358892)

Kohno S, Bernhard MC, Katsu Y, Zhu J, Bryan TA, Doheny BM, Iguchi T \& Guillette LJ Jr 2015 Estrogen receptor 1 (ESR1; ER $\alpha$ ), not ESR2 (ERß), modulates estrogen-induced sex reversal in the American alligator, a species with temperature-dependent sex determination. Endocrinology 156 1887-1899. (doi:10.1210/en.2014-1852)

Kratochvil L, Kubicka L \& Landova E 2008 Does the mechanism of sex determination constrain the potential for sex manipulation? A test in geckos with contrasting sex-determining systems Die Naturwissenschaften 95 209-215. (doi:10.1007/s00114-007-0317-0)

Lang JW \& Andrews HV 1994 Temperature-dependent sex determination in crocodilians. Journal of Experimental Zoology 270 28-44. (doi:10.1002/ jez.1402700105)

Matsumoto Y, Buemio A, Chu R, Vafaee M \& Crews D 2013 Epigenetic control of gonadal aromatase (cyp19a1) in temperature-dependent sex determination of red-eared slider turtles. PLOS ONE 8 e63599. (doi:10.1371/journal.pone.0063599)

McEwen BS, Lieberburg I, Chaptal C \& Krey LC 1977 Aromatization: important for sexual differentiation of the neonatal rat brain. Hormones and Behavior 9 249-263. (doi:10.1016/0018-506X(77)90060-5)

Meyer WJ III, Migeon BR \& Migeon CJ 1975 Locus on human $\mathrm{X}$ chromosome for dihydrotestosterone receptor and androgen insensitivity. PNAS 72 1469-1472. (doi:10.1073/pnas.72.4.1469)

Milnes MR, Roberts R \& Guillette LJ Jr 2002 Effects of incubation temperature and estrogen exposure on aromatase activity in the brain and gonads of embryonic alligators. Environmental Health Perspectives 110 (Suppl 3) 393-396. (doi:10.1289/ehp.02110s3393)

Mitchell NJ, Nelson NJ, Cree A, Pledger S, Keall SN \& Daugherty CH 2006 Support for a rare pattern of temperature-dependent sex determination in archaic reptiles: evidence from two species of tuatara (Sphenodon). Frontiers in Zoology 3 9. (doi:10.1186/1742-9994-3-9)

Morais da Silva S, Hacker A, Harley V, Goodfellow P, Swain A \& LovellBadge R 1996 Sox9 expression during gonadal development implies a conserved role for the gene in testis differentiation in mammals and birds. Nature Genetics 14 62-68. (doi:10.1038/ng0996-62) 
Mrosovsky N \& Pieau C 1991 Transitional range of temperature, pivotal temperatures and thermosensitive stages for sex determination in reptiles. Amphibia-Reptilia 12 169-179.

Nelson NJ, Moore JA, Pillai S \& Keall SN 2010 Thermosensitive period for sex determination in tuatara. Herpetological Conservation and Biology $\mathbf{5}$ 324-329.

Okada E, Yoshimoto S, Ikeda N, Kanda H, Tamura K, Shiba T, Takamatsu N \& Ito M 2009 Xenopus W-linked DM-W induces Foxl2 and Cyp19 expression during ovary formation. Sexual Development 3 38-42. (doi:10.1159/000200080)

Ottolenghi C, Pelosi E, Tran J, Colombino M, Douglass E, Nedorezov T, Cao A, Forabosco A \& Schlessinger D 2007 Loss of Wnt4 and Foxl2 leads to female-to-male sex reversal extending to germ cells. Human Molecular Genetics 16 2795-2804. (doi:10.1093/hmg/ddm235)

Pannetier M, Fabre S, Batista F, Kocer A, Renault L, Jolivet G, MandonPepin B, Cotinot C, Veitia R \& Pailhoux E 2006 FOXL2 activates P450 aromatase gene transcription: towards a better characterization of the early steps of mammalian ovarian development. Journal of Molecular Endocrinology 36 399-413. (doi:10.1677/jme.1.01947)

Parrott BB, Kohno S, Cloy-McCoy JA \& Guillette LJ Jr 2014 Differential incubation temperatures result in dimorphic DNA methylation patterning of the SOX9 and aromatase promoters in gonads of alligator (Alligator mississippiensis) embryos. Biology of Reproduction 90 2. (doi:10.1095/ biolreprod.113.111468)

Pieau C \& Dorizzi M 1981 Determination of temperature sensitive stages for sexual differentiation of the gonads in embryos of the turtle, Emy orbicularis. Journal of Morphology 170 373-382. (doi:10.1002/jmor. 1051700308)

Pieau C, Dorizzi M \& Richard-Mercier N 1999 Temperature-dependent sex determination and gonadal differentiation in reptiles. Cellular and Molecular Life Sciences 55 887-900. (doi:10.1007/s000180050342)
Pointer MA, Harrison PW, Wright AE \& Mank JE 2013 Masculinization of gene expression is associated with exaggeration of male sexual dimorphism. PLoS Genetics 9 e1003697. (doi:10.1371/journal.pgen.1003697)

Rhen T \& Lang JW 1998 Among-family variation for environmental sex determination in reptiles. Evolution 52 1514-1520. (doi:10.2307/2411322)

Shine R, Warner DA \& Radder R 2007 Windows of embryonic sexual lability in two lizard species with environmental sex determination. Ecology 88 1781-1788. (doi:10.1890/06-2024.1)

Smith CA \& Joss JMP 1993 Gonadal sex differentiation in Alligator mississippiensis, a species with temperature-dependent sex determination. Cell and Tissue Research 273 149-162. (doi:10.1007/BF00304622)

Tran D, Muesy-Dessole N \& Josso N 1977 Anti-Müllerian hormone is a functional marker of foetal Sertoli cells. Nature 269 411-412. (doi:10. 1038/269411a0)

Western PS, Harry JL, Graves JA \& Sinclair AH 1999a Temperature-dependent sex determination: upregulation of SOX9 expression after commitment to male development. Developmental Dynamics 214 171-177. (doi:10.1002/ (SICI)1097-0177(199903)214:3 <171::AID-AJA1 > 3.0.CO;2-S)

Western PS, Harry JL, Marshall Graves JA \& Sinclair AH $1999 b$ Temperaturedependent sex determination in the American alligator: $A M H$ precedes SOX9 expression. Developmental Dynamics 216 411-419. (doi:10.1002/ (SICI)1097-0177(199912)216:4/5<411::AID-DVDY9>3.0.CO;2-Y)

Wibbels T, Bull JJ \& Crews D 1991 Chronology and morphology of temperature-dependent sex determination. Journal of Experimental Zoology 260 371-381. (doi:10.1002/jez.1402600311)

Received 16 April 2015

First decision 11 May 2015

Revised manuscript received 2 July 2015

Accepted 16 July 2015 